\title{
Multicomponent Field Theories and Classical Rotators
}

\author{
François Dunlop \\ Inst. des Hautes Études Scientifiques, F-91 Bures-sur-Yvette, France \\ Charles M. Newman* \\ Dept. of Mathematics, Indiana University, Bloomington, Indiana 47401, USA
}

Received April 8, 1975

\begin{abstract}
It is shown that a $D$-component Euclidean quantum field, $\varphi=\left(\varphi^{1}, \ldots, \varphi^{D}\right)$, with $\lambda|\varphi|^{4}+\beta|\varphi|^{2}$ interaction, can be obtained as a limit of (ferromagnetic) classical rotator models; this extends a result of Simon and Griffiths from the case $D=1$. For these Euclidean field models, it is then shown that a Lee-Yang theorem applies for $D=2$ or 3 and that Griffiths' second inequality is valid for $D=2$; a complete proof is included of a Lee-Yang theorem for plane rotator and classical Heisenberg models. As an application of Griffiths' second inequality for $D=2$, an interesting relation between the "parallel" and "transverse" two-point correlations is obtained.
\end{abstract}

\section{Introduction}

Consider a multicomponent scalar field, $\varphi(x, t)=\left(\varphi^{1}(x, t), \ldots, \varphi^{D}(x, t)\right)$, in $d$-dimensional space-time with Hamiltonian,

$$
\int_{\mathbb{R}^{d-1}}\left[\frac{1}{2} \sum_{i=1}^{D}\left(\left(\pi^{i}\right)^{2}+\left|\nabla \varphi^{i}\right|^{2}+m_{0}^{2}\left(\varphi^{i}\right)^{2}\right)+\tilde{Q}\left(\sum_{i=1}^{D}\left(\varphi^{i}\right)^{2}\right)+\sum_{i=1}^{D} A^{i} \varphi^{i}\right] d x
$$

where $\pi=\partial \varphi / \partial t, \tilde{Q}$ is a polynomial with positive highest coefficient, and $\boldsymbol{A}(x, t)$ is an external field interacting with $\varphi$. The problem of constructing a corresponding quantum field has been considerably simplified in recent years by the probabilistic methods of Euclidean field theory (see, for example, the articles in [1]); it has been particularly realized that the associated Euclidean field is closely related (via a "lattice approximation") to certain models of ferromagnets from classical statistical mechanics [2].

The type of model we are concerned with consists of a family of random $D$-dimensional "spin" vectors $\left\{S_{j}=\left(S_{j}^{1}, \ldots, S_{j}^{D}\right): j=1, \ldots, N\right\}$ with joint probability distribution on $\left(\mathbb{R}^{D}\right)^{N}$,

$$
\frac{1}{Z} \exp \left(\sum_{j=1}^{N} a_{j} \cdot s_{j}+\sum_{j, k=1}^{N} \sum_{i=1}^{D} J_{j k}^{i} s_{j}^{i} s_{k}^{i}\right) \prod_{j=1}^{N} \varrho_{j}\left(s_{j}\right),
$$

where

$$
Z=Z\left(\left\{\boldsymbol{a}_{j}\right\},\left\{J_{j k}^{i}\right\}\right)=\int_{(\mathbb{R} D)^{N}} \exp \left(\sum_{j} \boldsymbol{a}_{j} \cdot \boldsymbol{s}_{j}+\sum_{j, k, i} J_{j k}^{i} s_{j}^{i} s_{k}^{i}\right) \prod_{j} d \varrho_{j}\left(\boldsymbol{s}_{j}\right),
$$

* Research supported in part by the National Science Foundation under grant NSF MPS 7404870 . 
and each $\varrho_{j}$ is a finite positive measure on $\mathbb{R}^{D}$. When the model arises from the Euclidean field determined by (1.1), the $J_{j k}^{i}$ are non-negative for $(j \neq k)$ and independent of $i$ while the $\varrho_{j}$ are given by

$$
d \varrho_{j}(s) / d s=\exp \left(-Q_{j}\left(|s|^{2}\right)\right),
$$

with $Q_{j}$ a polynomial related to $\tilde{Q}$ but altered by terms arising from the quadratic part of (1.1) and by counterterms needed for renormalization. We note in particular that in $|\varphi|^{4}$ models (i.e. $\tilde{Q}$ quadratic) with $d<5$, perturbation theory suggests that each $Q_{j}$ should be quadratic.

Statistical mechanical results have been applied to quantum field models primarily in the case $D=1[1,2]$. Certain results, such as the Griffiths-KellySherman inequalities, were proven directly (when $D=1$ ) for models given by (1.2)-(1.4) with arbitrary $Q_{j}$; other results, such as the Lee-Yang theorem require further approximation and do not apply to arbitrary $Q_{j}$ : Simon and Griffiths [3] showed (when $D=1$ ) that for $Q_{j}$ quadratic, (1.3) could be obtained as a certain limit of spin- $-\frac{1}{2}$ Ising models $\left(\varrho_{j}(s)=(\delta(s-1)+\delta(s+1)) / 2 \forall j\right)$ and consequently that the "classical" Lee-Yang theorem extends to these $\varphi^{4}$ models.

In Section 2 of this paper, we generalize the Simon-Griffiths result to $D>1$ by showing that $|\varphi|^{4}$ models can be obtained as limits of classical rotator models $\left(\varrho_{j}(s)=\delta\left(|s|^{2}-1\right) \forall j\right)$. Our proof is based on a multidimensional local limit theorem for large deviations [4] and is independent of $D$ (for $D>1$ ). While it is true that an ad hoc proof of our limit theorem can be given, it nevertheless seems more natural to place it in the general context of central limit theorems; indeed, the Simon-Griffiths result appears in this perspective as an application of Khintchine's classic results on large deviations for sums of independent Bernoulli random variables [5]. An added advantage is that in this approach analogues of the Euclidean field theory vertex functions arise in a quite interesting manner (see Remark 2 following Theorem 5 below).

Section 3 is devoted to the Lee-Yang theorem $(D=2$ or 3$)$. We begin with the results of Suzuki and Fisher for the quantum Heisenberg model [6] together with Lieb's analysis of the classical limit of quantum spin systems [7]; these yield a proof of the Lee-Yang theorem for the fully anisotropic classical Heisenberg model similar to the one sketched previously by Harris [8]. The plane rotator case then follows by adding an "infinite" self-coupling in a plane, and the $|\varphi|^{4}$ model $(D=2$ or 3) follows from our classical rotator approximation. In [9], Lee-Yang theorems for both plane rotators and classical Heisenberg models were announced, but proofs have not appeared and the hypotheses taken there seem too weak.

In Section 4, we obtain correlation inequalities $(D=2)$ by the methods of Ginibre [10]; these, together with our Lee-Yang theorem, show decay of the truncated two-point Schwinger functions for $|\varphi|^{4}$ models in a nonzero external field. We also prove that, in general two-component rotator models with a symmetry breaking field along the 1-component,

$$
E\left(S_{j}^{2} S_{k}^{2}\right)=O\left(\left[E\left(S_{j}^{1} S_{k}^{1}\right)-E\left(S_{j}^{1}\right) E\left(S_{k}^{1}\right)\right]^{1 / 2}\right) .
$$

This estimate implies that the "parallel" correlation length is no less than half the "transverse" correlation length or equivalently (for field theories) that the 
"parallel" mass gap is no more than twice the "transverse" mass gap. An explanation of this phenomenon in terms of the Goldstone picture is given at the end of Section 4; we note particularly that in a $\pi-\sigma$ model with spontaneously broken $O(2)$ symmetry (where $\varphi^{1}$ and $\varphi^{2}$ denote the $\sigma$ and $\pi$ fields respectively), our results do not contradict the possibility of having a zero mass pion and a non-zero mass $\sigma$ resonance. We mention finally that (1.5) may be used to compare the parallel and transverse susceptibilities; this has recently been done by Lebowitz and Penrose [11] who show, assuming the existence of spontaneous magnetization in a two-component model, that the parallel susceptibility diverges below the critical temperature in three or four spatial dimensions ${ }^{1}$.

\section{2. $|\varphi|^{4}$ Fields as Classical Rotators}

The main technical result of this section is the following theorem which generalizes [3, Theorem 1].

Theorem 1. Suppose $\left\{\boldsymbol{Y}_{k}: k=1,2, \ldots\right\}$ are independent random vectors uniformly distributed on the surface of the unit sphere in $\mathbb{R}^{D}$; then for any continuous complex valued function $F(\boldsymbol{y})$ on $\mathbb{R}^{D}$ which is bounded in modulus by $K \exp \left(c|\boldsymbol{y}|^{2}\right)$ for some $K$ and $c$, we have as $n \rightarrow \infty$

$$
A_{n} E\left(F(\boldsymbol{Y}(n)) \exp \left(b_{n}|\boldsymbol{Y}(n)|^{2}\right)\right) \rightarrow \int_{\mathbb{R}^{D}} F(\boldsymbol{y}) \exp \left(-\lambda|\boldsymbol{y}|^{4}\right) d \boldsymbol{y},
$$

where $\boldsymbol{Y}(n)=\left(\boldsymbol{Y}_{1}+\ldots+\boldsymbol{Y}_{n}\right) / n^{3 / 4}, A_{n}=\left(2 \pi / D n^{1 / 2}\right)^{D / 2}, b_{n}=n^{1 / 2} D / 2$, and $\lambda=D^{2} /(4 D+8)$.

The proof of this theorem is given below (following Theorem 5) for $D>1$ and is largely based on certain results of Richter concerning large deviations in the central limit theorem (Theorems 3 and 5). We do not include a proof for $D=1$, which could be analogously based on the classic results of Khintchine [5] for sums of Bernoulli random variables, since this has been given by Simon and Griffiths [3]; it is incidentally only when $D=1$ that $F$ need be assumed continuous. Before stating Richter's results, we give the following corollary of Theorem 1 which, together with Griffiths' method of "analogue spins" [12], will be used to obtain a Lee-Yang theorem for $|\varphi|^{4}$ models (see Theorem 10 below).

Theorem 2. Suppose $\left\{\boldsymbol{S}_{j}: j=1, \ldots, N\right\}$ are random $D$-dimensional vectors whose joint probability distribution is given by (1.2), (1.3), and (1.4), with $Q_{j}\left(s^{2}\right)=\lambda_{j} s^{4}+\beta_{j} s^{2}$ $\left(\lambda_{j}>0, \beta_{j} \in \mathbb{R}\right)$ and suppose that $\left\{\boldsymbol{Y}_{j, k}: j=1, \ldots, N ; k=1,2, \ldots\right\}$ are independent random vectors uniformly distributed on the surface of the unit sphere in $\mathbb{R}^{D}$; then (for $j=1, \ldots, N ; n=1,2, \ldots$ ) there exist constants $\tilde{A}_{n}>0, c_{j}>0$, and $b_{j, n} \rightarrow+\infty$ as $n \rightarrow \infty$, such that for any continuous complex valued function $F\left(s_{1}, \ldots, s_{N}\right)$ on $\left(\mathbb{R}^{D}\right)^{N}$ which is bounded in modulus by $K \exp \left(c\left(\left|s_{1}\right|^{2}+\ldots+\left|s_{N}\right|^{2}\right)\right)$ for some $K$ and $c$, we have

$$
\begin{aligned}
& \lim _{n \rightarrow \infty} \tilde{A}_{n} E\left(F\left(Y_{1}(n), \ldots, \boldsymbol{Y}_{N}(n)\right) \exp \left(\sum_{j} \boldsymbol{a}_{j} \cdot \boldsymbol{Y}_{j}(n)+\sum_{j, k, i}\left(J_{j k}^{i}+b_{j, n} \delta_{j k}\right) Y_{j}^{i}(n) Y_{k}^{i}(n)\right)\right) \\
& =E\left(F\left(\boldsymbol{S}_{1}, \ldots, \boldsymbol{S}_{N}\right)\right)
\end{aligned}
$$

where $\boldsymbol{Y}_{j}(n)=c_{j}\left(\boldsymbol{Y}_{j, 1}+\ldots+\boldsymbol{Y}_{j, n}\right) / n^{3 / 4}$ and $\delta_{j k}$ is Kronecker's delta.

Proof. This theorem follows immediately from Theorem 1 by standard arguments after choosing $c_{j}=\left(\lambda / \lambda_{j}\right)^{1 / 4}$ and $b_{j, n}=b_{n}\left(\lambda_{j} / \lambda\right)^{1 / 2}-\beta_{j}$.

\footnotetext{
1 An analogous result in field theory should follow from the Goldstone theorem.
} 
The next three theorems are not stated in their most general form. $\left\{\boldsymbol{Y}_{k}: k=1,2, \ldots\right\}$ will always be independent identically distributed $D$-dimensional random vectors whose common distribution is spherically symmetric and nontrivial $(\neq \delta(\boldsymbol{y}))$; it will also be assumed that

$$
\exp (G(\boldsymbol{a})) \equiv E\left(\exp \left(\boldsymbol{a} \cdot \boldsymbol{Y}_{1}\right)\right)<\infty \quad \forall \boldsymbol{a} \in \mathbb{R}^{D} .
$$

We define $\Gamma(\boldsymbol{y})$ for $\boldsymbol{y} \in \mathbb{R}^{D}$ (see [12]) by

$$
\Gamma(\boldsymbol{y})=\inf _{\boldsymbol{a} \in \mathbb{R}^{D}}(-\boldsymbol{a} \cdot \boldsymbol{y}+G(\boldsymbol{a})) .
$$

$\Gamma(y)$ is nonpositive and may take on the value $-\infty$ but it has a convergent (for small $|\boldsymbol{y}|)$ multi-Taylor series,

$$
\Gamma(\boldsymbol{y})=\sum_{m=1}^{\infty} \gamma_{2 m}|\boldsymbol{y}|^{2 m} /(2 m) !,
$$

which can be related to the Taylor series for $G$,

$$
G(\boldsymbol{a})=\sum_{m=1}^{\infty} u_{2 m}|\boldsymbol{a}|^{2 m} /(2 m) !,
$$

yielding in particular that

$$
\gamma_{2}=-1 / u_{2}, \quad \gamma_{4}=u_{4} /\left(u_{2}\right)^{4} \text {. }
$$

The following theorem is due to Richter [13]; it will be used in the proof of Theorem 1 to control the tail of the distribution of $Y(n)$.

Theorem 3. For $y>0$ and any $\boldsymbol{a} \in \mathbb{R}^{D}$,

$$
\operatorname{Pr}\left[\left(\boldsymbol{Y}_{1}+\ldots+\boldsymbol{Y}_{n}\right) \cdot \boldsymbol{a} \geqq y\right] \leqq \exp \left(n \Gamma\left(y \boldsymbol{a} / n|\boldsymbol{a}|^{2}\right)\right) \text {. }
$$

Proof. We let $Y=\left(\boldsymbol{Y}_{1}+\ldots+\boldsymbol{Y}_{n}\right) \cdot \boldsymbol{a}$ and rewrite Tchebyshev's inequality,

$$
\operatorname{Pr}\left[\exp (r Y) \geqq e^{w} E(\exp (r Y))\right] \leqq e^{-w},
$$

as

$$
\operatorname{Pr}[Y \geqq(w+n G(r \boldsymbol{a})) / r] \leqq e^{-w},
$$

for $r>0$. We choose $w=r y-n G(r \boldsymbol{a})$ in (2.9) and then choose $r$ so as to maximize $w$ which yields,

$$
\operatorname{Pr}[Y \geqq y] \leqq \exp \left(n \inf _{r>0}[-r y / n+G(r \boldsymbol{a})]\right) .
$$

Spherical symmetry now implies that (2.3) may be expressed as

$$
\Gamma(x)=\inf _{r>0}\left(-r \alpha|x|^{2}+G(r \alpha x)\right)
$$

for any $\alpha>0$; by choosing $\boldsymbol{x}=y \boldsymbol{a} / n|\boldsymbol{a}|^{2}$ and $\alpha=y / n|\boldsymbol{x}|^{2}=n|\boldsymbol{a}|^{2} / y$, we convert (2.10) into (2.7) as desired.

In order to see that the next theorem applies to classical rotators, we note that for $\boldsymbol{Y}_{1}$ uniformly distributed on the surface of the unit sphere in $\mathbb{R}^{D}$,

$$
\begin{aligned}
E\left(\exp \left(i \boldsymbol{a} \cdot \boldsymbol{Y}_{1}\right)\right) & =\text { const }|\boldsymbol{a}|^{1-D / 2} J_{(D / 2)-1}(|\boldsymbol{a}|) \\
& =\sum_{k=0}^{\infty} \frac{(-1)^{k}}{k ! D(D+2) \ldots(D+2 k-2)}\left(|a|^{2} / 2\right)^{k} ;
\end{aligned}
$$


here $J_{p}$ denotes the ordinary Bessel function of order $p$. It follows easily from (2.12) that $u_{2}=1 / D, \gamma_{2}=-D, u_{4}=-6 /\left(D^{3}+2 D^{2}\right), \gamma_{4}=-6 D^{2} / D+2$, and also that

$$
E\left(\exp \left(\boldsymbol{a} \cdot \boldsymbol{Y}_{1}\right)\right)<\exp \left(u_{2}|\boldsymbol{a}|^{2} / 2\right) \text { for }|\boldsymbol{a}| \neq 0 .
$$

Theorem 4. If $\boldsymbol{Y}_{1}$ satisfies (2.13), then $\Gamma(\boldsymbol{y})<\gamma_{2}|\boldsymbol{y}|^{2} / 2$ for $|\boldsymbol{y}| \neq 0$; if in addition $\boldsymbol{y}_{1}$ is bounded (i e. $\operatorname{Pr}\left[\left|\boldsymbol{y}_{1}\right|>k_{1}\right]=0$ for some $u_{1}$ ) and $y_{4}<0$, then there is a $y>0$ such that

$$
\Gamma(\boldsymbol{y}) \leqq\left(\gamma_{2}|\boldsymbol{y}|^{2} / 2\right)-\gamma|\boldsymbol{y}|^{4} \quad \forall \boldsymbol{y}
$$

and consequently there is a $C>0$ such that for $y>1$,

$$
\operatorname{Pr}\left[\left|\boldsymbol{Y}_{1}+\ldots+\boldsymbol{Y}_{n}\right| \geqq y\right] \leqq C y^{(D-1) / 2} \exp \left\{\left(\gamma_{2} y^{2} / 2 n\right)-\left(\gamma y^{4} / n^{3}\right)\right\} .
$$

Proof. If $\boldsymbol{Y}_{1}$ satisfies (2.13), then $G(\boldsymbol{a})<u_{2}|\boldsymbol{a}|^{2} / 2$ for $|\boldsymbol{a}| \neq 0$, so

$$
\Gamma(\boldsymbol{y})<\inf _{\boldsymbol{a}}\left(-\boldsymbol{a} \cdot \boldsymbol{y}+u_{2}|\boldsymbol{a}|^{2} / 2\right)=-|\boldsymbol{y}|^{2} / 2 u_{2}=\gamma_{2}|\boldsymbol{y}|^{2} / 2
$$

for $|\boldsymbol{y}| \neq 0$. We now suppose that $\boldsymbol{Y}_{1}$ is bounded and we define $h(\boldsymbol{y})=(\Gamma(\boldsymbol{y})-$ $\left.\gamma_{2}|\boldsymbol{y}|^{2} / 2\right) /|\boldsymbol{y}|^{4}$ for $|\boldsymbol{y}| \neq 0$ and denote by $K_{0}$ the essential supremum of $\left|\boldsymbol{Y}_{1}\right|$. Since $G(\boldsymbol{a}) \leqq K_{0}|\boldsymbol{a}|$ while $G(\boldsymbol{a}) /|\boldsymbol{a}| \rightarrow K_{0}$ as $|\boldsymbol{a}| \rightarrow \infty$, it can be easily seen that $\Gamma(\boldsymbol{y})=-\infty$ for $|\boldsymbol{y}|>K_{0}$ while $\Gamma(\boldsymbol{y})$ is continuous for $|\boldsymbol{y}|<K_{0}$; this implies that $h(\boldsymbol{y})$ is bounded away from zero for $|\boldsymbol{y}| \in[\varepsilon, \infty)$ for any $\varepsilon>0$. We may now obtain (2.14) by noting that since $\gamma_{4}<0, h(\boldsymbol{y})$ is bounded away from zero on $(0, \varepsilon)$ for small $\varepsilon$ by (2.4) so that $\gamma$ may be taken as $-\sup \{h(\boldsymbol{y}):|\boldsymbol{y}| \neq 0\}$.

It only remains to derive (2.15). We use (2.7), (2.14), and the straightforwardly proven geometric fact that there is a constant $C_{1}$ (depending only on $D$ ) such that there exist unit vectors $\left\{\boldsymbol{v}_{i}: i=1, \ldots, M \leqq C_{1} y^{(D-1) / 2}\right\}$ with the property that $\{\boldsymbol{y}:|\boldsymbol{y}| \geqq y\} \subset \bigcup_{i}\left\{\boldsymbol{y}: \boldsymbol{y} \cdot \boldsymbol{v}_{i} \geqq y-1\right\}$. Consequently,

$$
\operatorname{Pr}\left[\left|\boldsymbol{Y}_{1}+\ldots+\boldsymbol{Y}_{n}\right| \geqq y\right] \leqq C_{1} y^{(D-1) / 2} \exp \left\{\left(\gamma_{2}(y-1)^{2} / 2 n\right)-\left(\gamma(y-1)^{4} / n^{3}\right)\right\} ;
$$

since $\left|\boldsymbol{Y}_{1}+\ldots+\boldsymbol{Y}_{n}\right| \leqq K_{0} n$ with probability one, we may assume that $y \leqq K_{0} n$ in (2.17) which yields (2.15).

The following theorem is also due to Richter [4]; we do not include its complete proof which uses a saddle-point method but rather give a brief sketch in Remark 1 below. In order to see that its hypotheses are satisfied for classical rotators (when $D>1$ ), we note that for $\boldsymbol{Y}_{1}$ uniformly distributed on the surface of the unit sphere in $\mathbb{R}^{D}$, (2.12) implies that $\left|E\left(\exp \left(i \boldsymbol{a} \cdot \boldsymbol{Y}_{1}\right)\right)\right|=O\left(1 /|\boldsymbol{a}|^{(D-1) / 2}\right)$ for large $|\boldsymbol{a}|$ so that $E\left(\operatorname{expi}\left(\boldsymbol{a} \cdot \boldsymbol{Y}_{1}+\ldots+\boldsymbol{a} \cdot \boldsymbol{Y}_{n}\right)\right)$ is an $L^{1}$ function of $\boldsymbol{a}$ for $n>2 D /(D-1)$ which implies via an inverse Fourier transform that for $n>2 D /(D-1)$, the probability distribution of $\boldsymbol{Y}_{1}+\ldots+\boldsymbol{Y}_{n}$ has a bounded density.

Theorem 5. If there is an $n_{0}$ such that the probability distribution of $\boldsymbol{Y}_{1}+\ldots+\boldsymbol{Y}_{n}$ is absolutely continuous with bounded density $f_{n}(\boldsymbol{y})$ for $n>n_{0}$, then for $\sqrt{n}<|\boldsymbol{y}|=o(n)$ and for sufficiently large $n$,

$$
f_{n}(\boldsymbol{y})=\frac{\exp (n \Gamma(\boldsymbol{y} / n))}{\left(2 \pi n u^{2}\right)^{D / 2}}(1+O(|\boldsymbol{y}| / n)) .
$$


Remark 1. The Fourier transform of $f_{n}(\boldsymbol{y})$ is $(2 \pi)^{-D / 2} \exp (n G(i \boldsymbol{b}))$ so that by taking an inverse Fourier transform and changing the contour of integration, we have

$$
f_{n}(\boldsymbol{y})=(2 \pi)^{-D} \int \exp \left[-\left(\boldsymbol{a}_{0}+i \boldsymbol{b}\right) \cdot \boldsymbol{y}+n G\left(\boldsymbol{a}_{0}+i \boldsymbol{b}\right)\right] d \boldsymbol{b} .
$$

Richter chooses $\boldsymbol{a}_{0}$ so that the contour passes through a saddle-point and then estimates the integral asymptotically for large $n$; the value of the exponential at the saddle-point, which gives the principal part of the integral, may be evaluated as the minimum along the ridge:

$$
\exp \left[\inf _{\boldsymbol{a} \in \mathbb{R}^{D}}(-\boldsymbol{a} \cdot \boldsymbol{y}+n G(\boldsymbol{a}))\right]=\exp [n \Gamma(\boldsymbol{y} / n)] .
$$

For an alternate version of Theorem 5 which explains the relation of $\Gamma$ to entropy, see [14, Section A.4].

Remark 2. $\Gamma$ is a finite dimensional version of the generating function for vertex functions in Euclidean quantum field theory which is known to exist for certain $P(\varphi)_{2}$ models [15]. A version of Theorem 3 is then applicable (e.g. with $n=1)$ to relate the tail of the distribution of $P(\varphi)_{2}$ fields to the vertex functions; for $\varphi^{4}$ fields the first conclusion of Theorem 4 would also be applicable since an analogue of (2.13) has been shown to follow from the Lee-Yang theorem [16]. It would be quite interesting to obtain some generalization of Theorem 5 which applied to sums of independent Euclidean fields; such a result could be a useful new tool for studying vertex functions.

Proof of Theorem 1 ( for $D>1): \tilde{f}_{n}(\boldsymbol{y}) \equiv n^{3 D / 4} f_{n}\left(n^{3 / 4} \boldsymbol{y}\right)$ is the probability density of $\boldsymbol{Y}(n)$ so that the left hand side of $(2.1)$ is

$$
A_{n} \int_{\mathbb{R}^{D}} F(\boldsymbol{y}) \exp \left(b_{n}|\boldsymbol{y}|^{2}\right) \tilde{f}_{n}(\boldsymbol{y}) d \boldsymbol{y}=I_{1}(n)+I_{2}(n)+I_{3}(n),
$$

where $I_{1}, I_{2}, I_{3}$ are respectively obtained by restricting the region of integration on the left hand side of $(2.21)$ to $\left\{|\boldsymbol{y}| \leqq n^{-1 / 4}\right\},\left\{n^{-1 / 4}<|\boldsymbol{y}|<\alpha_{n}\right\},\left\{|\boldsymbol{y}| \geqq \alpha_{n}\right\}$ respectively; for reasons which are made clear below, we choose $\alpha_{n}$ so that $\left(\alpha_{n}\right)^{4} / \log n \rightarrow \infty$ while $\alpha_{n} / n^{1 / 12} \rightarrow 0$.

The assumed bound on $F$ implies that

$$
\begin{aligned}
\left|I_{1}(n)\right| & \leqq A_{n} K \int_{|\boldsymbol{y}| \leqq n^{-1 / 4}} \exp \left(\left(c+b_{n}\right)|\boldsymbol{y}|^{2}\right) \tilde{f}_{n}(\boldsymbol{y}) d \boldsymbol{y} \\
& \leqq\left(\mathrm{const} / n^{D / 4}\right) \int_{\mathbb{R}^{D}} \exp \left(c n^{-1 / 2}+1 / 2 D\right) \tilde{f}_{n}(\boldsymbol{y}) d y \rightarrow 0 \text { as } n \rightarrow \infty .
\end{aligned}
$$

We similarly have

$$
\left|I_{3}(n)\right| \leqq-\left(\text { const } / n^{D / 4}\right) \int_{\alpha_{n}}^{\infty} \exp \left(\left(c+b_{n}\right) y^{2}\right) d H(y),
$$

where $H(y)=\operatorname{Pr}[|\boldsymbol{Y}(n)|>y]$; integrating by parts and using the fact that $H(y)=0$ for $y>n^{1 / 4}$ yields

$$
\begin{aligned}
& \left|I_{3}(n)\right| \leqq\left(\text { const } / n^{D / 4}\right)\left(\exp \left(\left(c+b_{n}\right) \alpha_{n}^{2}\right) H\left(\alpha_{n}\right)+2\left(c+b_{n}\right)\right. \\
& \left.\cdot \int_{\alpha_{n}}^{\infty} y \exp \left(\left(c+b_{n}\right) y^{2}\right) H(y) d y\right) .
\end{aligned}
$$

Since $b_{n}=n^{1 / 2} \gamma_{2} / 2$, we have from (2.15) and the fact that $H(y)=0$ for $y>n^{1 / 4}$,

$$
\begin{aligned}
H(y) & \leqq C y^{(D-1) / 2} n^{3(D-1) / 8} \exp \left(-b_{n} y^{2}-\gamma y^{4}\right) \\
& \leqq C n^{(D-1) / 2} \exp \left(-b_{n} y^{2}-\gamma y^{4}\right)
\end{aligned}
$$


so that (2.24) implies that

$$
\begin{aligned}
\left|I_{3}(n)\right| & =O\left(n^{(D-2) / 4} \exp \left(c \alpha_{n}^{2}-\gamma \alpha_{n}^{4}\right)+n^{D / 4} \int_{\alpha_{n}}^{\infty} y \exp \left(c y^{2}-\gamma y^{4}\right) d y\right) \\
& =O\left(n^{D / 4} \exp \left(-\gamma \alpha_{n}^{4} / 2\right)\right) \rightarrow 0 \quad \text { as } \quad n \rightarrow \infty .
\end{aligned}
$$

It remains to show that $I_{2}(n)$ converges to the right hand side of $(2.1)$; it is here that Theorem 5 is used. We first scale (2.18) by $n^{3 / 4}$ and then use (2.4) to see that for $n^{-1 / 4}<|\boldsymbol{y}|<\alpha_{n}=o\left(n^{1 / 12}\right)=o\left(n^{1 / 4}\right)$,

$$
\begin{aligned}
\tilde{f}_{n}(y)= & \left(n^{1 / 2} / 2 \pi u_{2}\right)^{D / 2} \exp \left\{\left(n^{1 / 2} \gamma_{2}|\boldsymbol{y}|^{2} / 2\right)+\left(\gamma_{4}|\boldsymbol{y}|^{4} / 4 !\right)+O\left(|\boldsymbol{y}|^{6} / n^{1 / 2}\right)\right\} \\
& \cdot\left(1+O\left(|\boldsymbol{y}| / n^{1 / 4}\right)\right) \\
= & (1+O(1))\left(A_{n}\right)^{-1} \exp \left(-b_{n}|\boldsymbol{y}|^{2}-\lambda|\boldsymbol{y}|^{4}\right) .
\end{aligned}
$$

Consequently, it follows that as $n \rightarrow \infty$,

$$
I_{2}(n)=(1+o(1)) \int_{n^{-1 / 4}<|\boldsymbol{y}|<\alpha_{n}} F(\boldsymbol{y}) e^{-\lambda|\boldsymbol{y}|^{4}} d \boldsymbol{y} \rightarrow \int_{\mathbb{R}^{D}} F(\boldsymbol{y}) e^{-\lambda|\boldsymbol{y}|^{4}} d \boldsymbol{y}
$$

which completes the proof of Theorem 1.

Remark 3. It is clear from the above proof that the conclusions of Theorem 1 remain valid for $Y_{j}$ 's other than classical rotators (with appropriate changes of $\left.A_{n}, b_{n}, \lambda\right)$ providing the hypotheses of Theorems 4 and 5 are satisfied. This fact seems to endow $|\varphi|^{4}$ models with a kind of universality which should perhaps be further investigated.

\section{Zeros of Partition Functions}

The primary purpose of this section is to obtain a Lee-Yang theorem for $|\varphi|^{4}$ models and classical rotators with $D=2$ or 3 . We include a complete proof for the classical case since, as explained in the introduction, the present status of Lee-Yang theorems for these models is somewhat unclear.

We define the partition function for a classical Heisenberg model as

$$
Z^{C}\left(\left\{\boldsymbol{a}_{j}\right\},\left\{J_{j k}^{i}\right\}\right)=E\left(\exp \left(\sum_{j=1}^{N} \boldsymbol{a}_{j} \cdot \boldsymbol{Y}_{j}+\sum_{j, k=1}^{N} \sum_{i=1}^{3} J_{j k}^{i} Y_{j}^{i} Y_{k}^{i}\right)\right)
$$

where $\left\{\boldsymbol{Y}_{j}: j=1, \ldots, N\right\}$ are independent 3-dimensional random vectors uniformly distributed on the surface of the unit sphere in $\mathbb{R}^{3}$. Our analysis of the zeros of $Z^{C}$ is based on the following theorem of Suzuki and Fisher [6] concerning the partition function $Z^{Q}$ of a quantum Heisenberg model of $\operatorname{spin} \mathscr{J}(\mathscr{J}=1 / 2,1,3 / 2, \ldots)$. $Z^{Q}$ is defined as

$$
Z^{Q}\left(\left\{\boldsymbol{a}_{j}\right\},\left\{J_{j k}^{i}\right\}, \mathscr{J}\right)=(2 \mathscr{J}+1)^{-N} \operatorname{Tr}\left(\exp \left(\sum_{j=1}^{N} \boldsymbol{a}_{j} \cdot \boldsymbol{\sigma}_{j}+\sum_{j, k=1}^{N} \sum_{i=1}^{3} J_{j k}^{i} \sigma_{j}^{i} \sigma_{k}^{i}\right)\right),
$$

where $\left\{\sigma_{j}=\left(\sigma_{j}^{1}, \sigma_{j}^{2}, \sigma_{j}^{3}\right): j=1, \ldots, N\right\}$ are standard quantum mechanical spin operators satisfyibg: $\left[\sigma_{j}, \sigma_{k}\right]=0$ for $j \neq k,\left[\sigma_{j}^{1}, \sigma_{j}^{2}\right]=i \sigma_{j}^{3}$ (and cyclically), and $\left(\sigma_{j}^{1}\right)^{2}+\left(\sigma_{j}^{2}\right)^{2}+\left(\sigma_{j}^{3}\right)^{2}=\mathscr{J}(\mathscr{J}+1)$; these operators act on the tensor product $\bigotimes_{j=1}^{N} \mathbb{C}^{2 \mathscr{f}+1}$ in the usual way [7].

Theorem 6. If $\left|J_{j k}^{2}\right| \leqq J_{j k}^{1},\left|J_{j k}^{3}\right| \leqq J_{j k}^{1}$, and $\boldsymbol{a}_{j}=\left(a_{j}, 0,0\right)$ with $a_{j} \geqq 0$ for all $j, k$; then for any $\mathscr{J}$,

$$
Z^{Q}(z) \equiv Z^{Q}\left(\left\{z \boldsymbol{a}_{j}\right\},\left\{J_{j k}^{i}\right\}, \mathscr{J}\right)
$$

is an entire function of $z$ all of whose zeros are pure imaginary. 
Proof. This theorem is basically given by Suzuki and Fisher in [6]; we only note here that their proof allows $J_{k k}^{i} \neq 0$ since they reduce the general spin $\mathscr{J}$ situation to the $\mathscr{J}=1 / 2$ case where $\left(\sigma^{1}\right)^{2}=\left(\sigma^{2}\right)^{2}=\left(\sigma^{3}\right)^{2}=1 / 4$.

The next theorem is due to Lieb [7].

Theorem 7. For any choice of $\left\{\boldsymbol{a}_{j}\right\},\left\{J_{j k}^{i}\right\}$,

$$
\underline{A} Z^{C}\left(\left\{\mathscr{J} \boldsymbol{a}_{j}\right\},\left\{J_{j k}^{i}\right\}\right) \leqq Z^{Q}\left(\left\{J_{j k}^{i}\right\},\left\{\boldsymbol{a}_{j}\right\}, \mathscr{J}\right) \leqq \bar{A} Z^{C}\left(\left\{(\mathscr{J}+1) \boldsymbol{a}_{j}\right\},\left\{\bar{J}_{j k}^{i}\right\}\right)
$$

where

$$
\begin{aligned}
\underline{J}_{j k}^{i} & =\left(\mathscr{J}^{2}-\mathscr{J} \delta_{j k} / 2\right) J_{j k}^{i}, \\
\bar{J}_{j k}^{i} & =\left((\mathscr{J}+1)^{2}+(\mathscr{J}+1) \delta_{j k} / 2\right) J_{j k}^{i}, \\
A & =\exp \left(\sum_{k, i} \mathscr{J} J_{k k}^{i} / 2\right), \\
\bar{A} & =\exp \left(-\sum_{k, i}(\mathscr{J}+1) J_{k k}^{i} / 2\right) .
\end{aligned}
$$

Consequently,

$$
Z^{C}\left(\left\{\boldsymbol{a}_{j}\right\},\left\{J_{j k}^{i}\right\}\right)=\lim _{\mathscr{J} \rightarrow \infty} Z^{Q}\left(\left\{\boldsymbol{a}_{j} / \mathscr{J}\right\},\left\{J_{j k}^{i} / \mathscr{J}^{2}\right\}, \mathscr{J}\right) .
$$

Proof. These results are all contained in [7]; we include a statement of Lieb's inequalities to make clear that (3.9) is still valid with $J_{k k}^{i} \neq 0$.

We can now prove a Lee-Yang Theorem for classical Heisenberg models.

Theorem 8. If $\left|J_{j k}^{2}\right| \leqq J_{j k}^{1},\left|J_{j k}^{3}\right| \leqq J_{j k}^{1}$, and $\boldsymbol{a}_{j}=\left(a_{j}, 0,0\right)$ with $a_{j} \geqq 0$ for all $j, k$; then

$$
Z^{C}(z) \equiv Z^{C}\left(\left\{z a_{j}\right\},\left\{J_{j k}^{i}\right\}\right)
$$

is an entire function of $z$ all of whose zeros are pure imaginary.

Proof. We define $Z_{\mathscr{f}}^{Q}(z)$ to be the right hand side of (3.3) with $a_{j}$ replaced by $\boldsymbol{a}_{j} / \mathscr{J}$ and $J_{j k}^{i}$ replaced by $J_{j k}^{i} / \mathscr{J}^{2}$ for all $j, k, i$. Now, $Z_{\mathscr{g}}^{Q}(z) \rightarrow Z^{C}(z)$ as $\mathscr{J} \rightarrow \infty$ uniformly on compact subsets of real $z$ by Theorem 7. Moreover, the normalized trace [in (3.2)] of a matrix is bounded by the matrix norm, and $\left\|\sigma_{j}^{i} / \mathscr{J}\right\| \leqq 1$, so that $Z_{\bar{f}}^{Q}(z)$ is uniformly bounded on compact subsets of complex $z$ (a more elegant argument actually shows that $\left|Z_{\mathscr{F}}^{Q}(z)\right| \leqq Z_{\mathscr{F}}^{Q}(\operatorname{Re} z)$; this is based on the fact that $\mid(\operatorname{Tr}(\exp (A+i B)) \mid \leqq \operatorname{Tr}(\exp (A))$ for self-adjoint $A$ and $B)$. The Vitali convergence theorem thus implies that $Z_{\mathscr{G}}^{Q} \rightarrow Z^{C}$ as $\mathscr{J} \rightarrow \infty$ uniformly on compact subsets of complex $z$. Theorem 6 together with Hurwitz' Theorem [17, p. 205] then implies that the zeros of $Z^{C}$ are all pure imaginary as desired.

As a corollary to Theorem 8 , we obtain a Lee-Yang theorem for plane rotators:

Theorem 9. Suppose $\left\{\boldsymbol{X}_{j}: j=1, \ldots, N\right\}$ are independent 2-dimensional random vectors uniformly distributed on the unit circle in $\mathbb{R}^{2}$, and

$$
Z(z)=E\left(\exp \left(z \sum_{j=1}^{N} a_{j} X_{j}^{1}+\sum_{j, k=1}^{N} \sum_{i=1}^{2} J_{j k}^{i} X_{j}^{i} X_{k}^{i}\right)\right)
$$

with $a_{j} \geqq 0$ and $\left|J_{j k}^{2}\right| \leqq J_{j k}^{1}$ for all $j, k$; then $Z(z)$ is an entire function of $z$ all of whose zeros are pure imaginary.

Proof. Given $\left\{a_{j}\right\},\left\{J_{j k}^{1}\right\},\left\{J_{j k}^{2}\right\}$, we define

$$
Z_{n}(z)=B_{n} Z^{C}\left(\left\{z \boldsymbol{a}_{j}\right\},\left\{J_{j k}^{i}(n)\right\}\right),
$$


with $\boldsymbol{a}_{j}=\left(a_{j}, 0,0\right), J_{j k}^{i}(n)=J_{j k}^{i}+n \delta_{j k}(i=1,2), J_{j k}^{3}(n)=0$, and

$$
B_{n}=\left(E\left[\exp \left\{n \sum_{j=1}^{N}\left(\left(Y_{j}^{1}\right)^{2}+\left(Y_{j}^{2}\right)^{2}\right)\right\}\right]\right)^{-1},
$$

where $\left\{\boldsymbol{Y}_{j}\right\}$ are independent 3-dimensional rotators. Since $\left(Y_{j}^{1}\right)^{2}+\left(Y_{j}^{2}\right)^{2}=1-\left(Y_{j}^{3}\right)^{2}$, we may use the fact that for suitably chosen $\tilde{B}_{n}$,

$$
\tilde{B}_{n} \exp \left(-n\left(y_{3}\right)^{2}\right) \delta\left(y_{1}^{2}+y_{2}^{2}+y_{3}^{2}-1\right) \rightarrow \delta\left(y_{1}^{2}+y_{2}^{2}-1\right) \delta\left(y_{3}\right)
$$

to conclude that $Z_{n} \rightarrow Z$ uniformly on compact subsets of $\mathbb{C}$. By Theorem 8 , $Z_{n}$ has only pure imaginary zeros so that by Hurwitz' theorem $Z$ has only pure imaginary zeros; this completes the proof.

Our final result is a Lee-Yang theorem for $|\varphi|^{4}$ models $(D=2$ or 3$)$.

Theorem 10. Let $Z(z)=Z\left(\left\{z \boldsymbol{a}_{j}\right\},\left\{J_{j k}^{i}\right\}\right)$ be defined by (1.3) and (1.4) with $Q_{j}\left(s^{2}\right)=$ $\lambda_{j} s^{4}+\beta_{j} s^{2}\left(\lambda_{j}>0, \beta_{j} \in \mathbb{R}\right)$ and $D=2$ or 3 . Suppose further that $a_{j}^{1} \geqq 0, a_{j}^{i}=0(i \neq 1)$, $J_{j k}^{1} \geqq\left|J_{j k}^{i}\right|(i \neq 1, j \neq k)$, and $J_{k k}^{1} \geqq J_{k k}^{i}(i \neq 1)$; then $Z(z)$ is an entire function of $z$ all of whose zeros are pure imaginary.

Proof. This theorem follows immediately from Theorems 2,8 , and 9, via standard arguments.

Remark 4. The reason we have only been able to obtain results on zeros of the partition function (Theorems 8,9, and 10) for those nonquantum models where the spin dimension $D$ satisfies $D \leqq 3$, is that our proofs are all based on the quantum model result $(D=3)$ of Suzuki-Fisher (Theorem 6). There seem to be good reasons why analogous Lee-Yang theorems should apply to arbitrary spin dimension; in fact, Eq. (2.12) shows that for a single $D$-dimensional classical rotator $\boldsymbol{Y}, \exp \left(z Y^{1}\right)$ has only pure imaginary zeros so that the results of [18] imply a weak Lee-Yang theorem (valid under the extra assumption that $J_{j k}^{i}=0$ for $i \neq 1$ ). In order to obtain a stronger result for $D>3$, and in view of the fact that the Suzuki-Fisher theorem is itself based on a classical spin- $\frac{1}{2}$ approximation, it would appear reasonable to search for a direct spin- $\frac{1}{2}$ approximation to $D$-dimensional classical rotators (or $|\varphi|^{4}$ models); such a result would have the additional advantage of eliminating the need for much of the material of this section.

\section{Correlation Inequalities}

The first theorem of this section states a version of the first inequality of Griffiths, Kelly, and Sherman which is appropriate for $D$-spin dimensional ferromagnets; its proof follows simply from the methods developed in $[19,10,2]$. When $a^{j} \geqq 0 \forall j$ we write $\boldsymbol{a} \geqq 0$ and for a multi-index $\boldsymbol{n}=\left(n^{1}, \ldots, n^{D}\right)$ we denote $\left(S^{1}\right)^{n^{1}} \ldots\left(S^{D}\right)^{n^{D}}$ by $S^{n}$; we say $\varrho(s)$ is reflection invariant if for each $k$ it is invariant under $\left(s^{1}, \ldots, s^{k}, \ldots, s^{D}\right) \rightarrow\left(s^{1}, \ldots,-s^{k}, \ldots, s^{D}\right)$.

Theorem 11. Suppose $\left\{\boldsymbol{S}_{j}: j=1, \ldots, N\right\}$ are random D-dimensional vectors whose joint probability distribution is given by (1.2) and (1.3) with each $\varrho_{j}$ reflection invariant and such that $\int \exp \left(b|\mathbf{s}|^{2}\right) d \varrho_{j}(\mathbf{s})<\infty \forall b>0$; if $\boldsymbol{a}_{j} \geqq 0$ and $J_{j k}^{i} \geqq 0 \forall j, k, i(j \neq k)$, then for any $\boldsymbol{n}_{1}, \ldots, \boldsymbol{n}_{N}$,

$$
E\left(\boldsymbol{S}_{1}^{n_{1}} \ldots S_{N}^{n_{N}}\right) \geqq 0 .
$$


Proof. It suffices to show that for any choice of $\left\{\boldsymbol{n}_{j}\right\}$,

$$
\int \boldsymbol{s}_{1}^{\boldsymbol{n}_{1}} \ldots \boldsymbol{s}_{N}^{\boldsymbol{n}_{N}} \exp \left(\sum_{j, i} a_{j}^{i} s_{j}+\sum_{j, k, i} J_{j k}^{i} s_{j}^{i} s_{k}^{i}\right) \prod_{j=1}^{N} d \varrho_{j}\left(\boldsymbol{s}_{j}\right) \geqq 0 .
$$

We may assume, without loss of generality, that $J_{k k}^{i} \equiv 0$, since these terms could in any case be absorbed into the $\varrho_{k}$ 's. Expanding the exponential factor in (4.2) as an infinite series gives a multi-Taylor series for (4.2) in powers of $\left\{a_{j}^{i}, J_{j k}^{i}\right\}$ each coefficient of which is expressible as a product of factors of the form

$$
\int_{\mathbb{R}^{D}} s_{j}^{m_{j}} d \varrho_{j}\left(s_{j}\right)
$$

for some multi-index $\boldsymbol{m}_{j}$. Since $a_{j}^{i}, J_{j k}^{i} \geqq 0$, we need only show that the expression (4.3) is non-negative; this follows from the reflection invariance of $\varrho_{j}$ which implies that (4.3) vanishes unless $m_{j}^{i}$ is even for all $i$.

In contrast to the previous theorem, Griffiths' second inequality has not been extended to multicomponent ferromagnets in any generality; it is not even known whether the isotropic classical Heisenberg model (classical rotator with $D=3$ ) satisfies any inequality of this type. However, for $D=2$, Ginibre's results for plane rotators can be extended to $Q\left(|\varphi|^{2}\right)$ models. We first state two lemmas basically corresponding to examples given by Ginibre in his general formulation of Griffiths' inequalities (see [10] for details).

Lemma 1. Let $\mathscr{F}$ be the set of multinomials in $\left\{\cos \left(m_{1} \theta_{1}+\ldots+m_{N} \theta_{N}\right): m_{i} \in \mathbb{Z}\right\}$ with non-negative coefficients; then for any finite family $\left\{f_{1}, \ldots, f_{n}\right\}$ of elements of $\mathscr{F}$ and any sequence of plus or minus signs,

$$
\int_{0}^{2 \pi} \ldots \int_{0}^{2 \pi} \prod_{j=1}^{N} d \theta_{j} d \theta_{j}^{\prime} \prod_{i=1}^{n}\left(f_{i}\left(\theta_{1}, \ldots, \theta_{N}\right) \pm f_{i}\left(\theta_{1}^{\prime}, \ldots, \theta_{N}^{\prime}\right)\right) \geqq 0 .
$$

Lemma 2. Let $\mathscr{G}$ be the set of multinomials in $\left\{\prod_{j=1}^{N} h_{j}\left(r_{j}\right)\right.$ : each $h_{j}(r)$ is nonnegative, nondecreasing on $[0, \infty)$ and $O\left(\exp \left(b r^{2}\right)\right)$ for some $\left.b>0\right\}$ with non-negative coefficients; then for any positive measures $v_{j}(r)(j=1, \ldots, N)$ such that $\int_{0}^{\infty} \exp \left(b r^{2}\right)$. $d v_{j}(r)<\infty \forall b, j$ and any finite family $\left\{g_{1}, \ldots, g_{n}\right\}$ of elements of $\mathscr{G}$ and any sequence of plus or minus signs,

$$
\int_{0}^{\infty} \ldots \int_{0}^{\infty} \prod_{j=1}^{N} d v_{j}\left(r_{j}\right) d v_{j}\left(r_{j}^{\prime}\right) \prod_{i=1}^{n}\left(g_{i}\left(r_{1}, \ldots, r_{N}\right) \pm g_{i}\left(r_{1}^{\prime}, \ldots, r_{N}^{\prime}\right)\right) \geqq 0 .
$$

Remark 5. In [10], the analogue of Lemma 1 for the group $\mathbb{Z}_{2}$ (spin $\frac{1}{2}$ ) and Lemma 2 were used to prove the GKS inequalities for continuous one-dimensional spins. We proceed to apply the same method for $D=2$.

We define $\mathscr{2}$ as the family of functions on $\left(\mathbb{R}^{2}\right)^{N}$ which (in polar coordinates) are multinomials of functions from $\mathscr{F}$ and $\mathscr{G}$ with non-negative coefficients.

Theorem 12. Suppose $\left\{S_{j}: j=1, \ldots, N\right\}$ are random 2-dimensional vectors whose joint probability distribution is given by (1.2) and (1.3) with each $d \varrho_{j}(s)=d \varrho_{j}(\theta, r)=$ $d \theta_{j} d v_{j}(r)$ and such that $\int \exp \left(b|s|^{2}\right) d \varrho_{j}(\mathbf{s})<\infty \forall b, j$; if $\boldsymbol{a}_{j}=\left(a_{j}, 0\right) \geqq 0,\left|J_{j k}^{2}\right| \leqq J_{j k}^{1}(j \neq k)$, and $J_{k k}^{2} \leqq J_{k k}^{1}$ for all $j, k$, then for any $F, G \in \mathscr{Q}$,

$$
\begin{aligned}
& E\left(F\left(\boldsymbol{S}_{1}, \ldots, \boldsymbol{S}_{N}\right)\right) \geqq 0, \\
& E\left(F\left(\boldsymbol{S}_{1}, \ldots, \boldsymbol{S}_{N}\right) G\left(\boldsymbol{S}_{1}, \ldots, \boldsymbol{S}_{N}\right)\right) \geqq E\left(F\left(\boldsymbol{S}_{1}, \ldots, \boldsymbol{S}_{N}\right)\right) E\left(G\left(\boldsymbol{S}_{1}, \ldots, \boldsymbol{S}_{N}\right)\right) .
\end{aligned}
$$


Proof. We first note that, by absorbing the appropriate factor into $v_{k}(r)$, we may assume, without loss of generality, that $\left|J_{k k}^{2}\right| \leqq J_{k k}^{1}$. Next we notice that for arbitrary $j, k$,

$$
\begin{aligned}
J_{j k}^{1} s_{j}^{1} s_{k}^{1}+J_{j k}^{2} s_{j}^{2} s_{k}^{2}= & r_{j} r_{k}\left\{J_{j k}^{1}-\left|J_{j k}^{2}\right|\right) \cos \theta_{1} \cos \theta_{2} \\
& \left.+\left|J_{j k}^{2}\right| \cos \left(\theta_{1}-\varepsilon_{j k} \theta_{2}\right)\right\} \in \mathscr{Q},
\end{aligned}
$$

where $\varepsilon_{j k}=\operatorname{sgn}\left(J_{j k}^{2}\right)$. Also, $\boldsymbol{a}_{j} \cdot \boldsymbol{s}_{j}=a_{j} s_{j}^{1}=a_{j} r_{j} \cos \theta_{j} \in \mathscr{Q}$. The theorem now follows from [10, Proposition 3].

Remark 6. For any choice of $n_{1}, \ldots, n_{N}, \prod_{j=1}^{N}\left(s_{j}^{1}\right)^{n_{j}} \in \mathcal{Q}$, but in general $\prod_{j=1}^{N}\left(\boldsymbol{s}_{j}\right)^{\boldsymbol{n}_{j}}$ does not belong to 2 . Although Theorem 12 only proves monotonicity in the thermodynamic limit for expectations of functions in $\mathscr{Q}$, this is still sufficient to imply uniqueness of the thermodynamic limit (i.e., independent of the shape of the finite volume regions but possibly dependent on boundary conditions) ${ }^{2}$. We note that some new correlation inequalities related to those of Theorem 12 have recently been obtained $[20,21]$.

Remark 7. One important application of the Lee-Yang theorem and GKS inequalities for $D=1$ was the decay of the truncated two-point Schwinger function for $\lambda \varphi^{4}+\beta \varphi^{2}-a \varphi$ models $(a \neq 0)$ [22, Section IX.4] which in turn implies uniqueness of the vacuum. The same methods, when combined with Theorems 10 and 12 , can be used for $D=2$ to yield that for $\lambda\left(\left(\varphi^{1}\right)^{2}+\left(\varphi^{2}\right)^{2}\right)^{2}+\beta\left(\left(\varphi^{1}\right)^{2}+\right.$ $\left.\left(\varphi^{2}\right)^{2}\right)-a \varphi^{1}$ models $(a \neq 0), E\left(\varphi^{1}(x) \varphi^{1}\left(x^{\prime}\right)\right)-E\left(\varphi^{1}(x)\right) E\left(\varphi^{1}\left(x^{\prime}\right)\right) \rightarrow 0$ as $\left|x-x^{\prime}\right| \rightarrow \infty$. The $\varphi^{2} \rightarrow-\varphi^{2}$ symmetry for such a model shows that $E\left(\varphi^{1}(x) \varphi^{2}\left(x^{\prime}\right)\right)=0=E\left(\varphi^{2}(x)\right)$, so that the only other non-zero two-point function to investigate is $E\left(\varphi^{2}(x) \varphi^{2}\left(x^{\prime}\right)\right)$ $E\left(\varphi^{2}(x)\right) E\left(\varphi^{2}\left(x^{\prime}\right)\right)=E\left(\varphi^{2}(x) \varphi^{2}\left(x^{\prime}\right)\right)$; the following theorem shows that this "transverse" two-point function decays at least as fast as the square root of the "parallel" truncated two point function.

Theorem 13. Suppose $\left\{\boldsymbol{S}_{j}: j=1, \ldots, N\right\}$ are random 2-dimensional vectors satisfying the hypotheses of Theorem 12; then

$$
\left[E\left(S_{j}^{2} S_{k}^{2}\right)\right]^{2} \leqq\left[E\left(S_{j}^{1} S_{k}^{1}\right)-E\left(S_{j}^{1}\right) E\left(S_{k}^{1}\right)\right] \cdot\left[E\left(S_{j}^{1} S_{k}^{1}\right)+E\left(S_{j}^{1}\right) E\left(S_{k}^{1}\right)\right] .
$$

Proof. Following [10], we use the Ginibre-Percus method of introducing an independent identically distributed duplicate system $\left\{\boldsymbol{S}_{j}^{\prime}: j=1, \ldots, N\right\}$. Then, using polar coordinates, we have from Theorem 12,

$$
E\left(r_{j} r_{j}^{\prime} \cos \left(\theta_{j}+\theta_{j}^{\prime}\right) r_{k} r_{k}^{\prime} \cos \left(\theta_{k}-\theta_{k}^{\prime}\right)\right) \geqq E\left(r_{j} r_{j}^{\prime} \cos \left(\theta_{j}+\theta_{j}^{\prime}\right)\right) E\left(r_{k} r_{k}^{\prime} \cos \left(\theta_{k}-\theta_{k}^{\prime}\right)\right) .
$$

Expanding the cosines, factoring the expectations via the independence of the two systems, and using the fact that (by the symmetry $S_{j}^{2} \rightarrow-S_{j}^{2}$ or $\theta_{j} \rightarrow-\theta_{j} \forall j$ )

$$
E\left(S_{j}^{2}\right)=E\left(S_{k}^{2}\right)=E\left(S_{j}^{1} S_{k}^{2}\right)=E\left(S_{j}^{2} S_{k}^{1}\right)=0 \quad \forall j, k,
$$

yields

$$
\left[E\left(S_{j}^{1} S_{k}^{1}\right)\right]^{2}-\left[E\left(S_{j}^{2} S_{k}^{2}\right)\right]^{2} \geqq\left[E\left(S_{j}^{1}\right)\right]^{2}\left[E\left(S_{k}^{1}\right)\right]^{2}
$$

which gives (4.9) as desired.

If the "parallel" truncated two-point function decays exponentially, then Theorem 13 implies that the "transverse" two-point function does so as well with

\footnotetext{
${ }^{2}$ For example $S_{l}^{2} S_{J}^{2}=1 / 2 \pi_{i} \pi_{j}\left(\cos \left(\theta_{l}-\theta_{j}\right)-\cos \left(\theta_{\imath}+\theta_{j}\right)\right)$.
} 
correlation length no greater than twice the "parallel" correlation length; for a 2-component field theory model, this means that the "transverse" mass gap is at least half the "parallel" mass gap (in the presence of a non-zero external field which defines the parallel and transverse directions).

To compare these mass inequalities with the Goldstone picture of broken symmetry, we consider a $\pi-\sigma$ model with spontaneously broken $O(2)$ symmetry where $\varphi^{1}$ and $\varphi^{2}$ of (1.1) respectively denote the $\sigma$ and $\pi$ fields. Spontaneously broken symmetry corresponds to the choice of a pure phase (with zero external field) in the Euclidean world in which $E\left(\varphi^{1}\right)=M>0$ while $E\left(\varphi^{2}\right)=0$; the pion is thus to be the Goldstone boson of this model. For simplicity we consider a $(\varphi \cdot \varphi)^{2}$ model with effective potential,

$$
V(\varphi)=A(\varphi \cdot \varphi)^{2}-B(\varphi \cdot \varphi)
$$

and with $A, B>0$. A minimum of $V\left(\varphi^{1}, 0\right)$ occurs at $\varphi^{1}=(B / 2 A)^{1 / 2}$ which is thus the Goldstone value of $M$; with this choice of $M$, we let $\boldsymbol{\theta}=\boldsymbol{\varphi}-(M, 0)$ and rewrite $V$ as

$$
\tilde{V}(\boldsymbol{\theta}) \equiv V(\boldsymbol{\theta}+(M, 0))=\tilde{V}_{0}(\boldsymbol{\theta})+\tilde{V}_{1}(\boldsymbol{\theta})
$$

where

$$
\tilde{V}_{0}(\theta)=4 M^{2} A\left[\theta^{1}\right]^{2}+\text { const }
$$

and

$$
\tilde{V}_{1}(\boldsymbol{\theta})=4 A M \theta^{1}\left[\theta^{2}\right]^{2}+4 A M\left[\theta^{1}\right]^{3}+A(\boldsymbol{\theta} \cdot \boldsymbol{\theta})^{2} .
$$

Now in the simplest version of the Goldstone picture, one disregards $\tilde{V}_{1}$ and, based on the fact that the coefficient of $\left[\theta^{2}\right]^{2}$ in $\tilde{V}_{0}$ is zero, concludes that the pion has zero mass while the " $\sigma$-field particle" has positive mass. This explanation appears to be in direct contradiction with Theorem 13 which implies that whenever the $\pi$-field mass gap vanishes, so must the $\sigma$-field mass gap; to remove the contradiction and also to indicate that the square root of Theorem 13 is optimal ${ }^{3}$ we follow an analysis due to Coleman [23] by considering $\tilde{V}_{1}$.

The point is that the term $4 M \theta^{1}\left[\theta^{2}\right]^{2}$ in $\tilde{V}_{1}$ couples the $\theta^{1}$ and $\theta^{2}$ fields in such a way that what we formerly regarded as a $\sigma$-particle with positive mass is now changed into a $\sigma$-resonance (with positive mass). Moreover, since the state of $\theta^{1}$ applied to the vacuum is now coupled to the state of $\left[\theta^{2}\right]^{2}$ applied to the vacuum, the $\sigma$-resonance should decay into two (zero-mass) pions; this, together with the fact that the $\sigma$-resonance cannot decay into one pion by the $\theta^{2} \rightarrow-\theta^{2}$ symmetry ( $G$-parity), indicates that the square root of Theorem 13 gives the correct relation between transverse $(\pi)$ and parallel $(\sigma)$ two-point functions. We note however that in the 4-state Potts model (classical "rotator" with discrete values $\theta_{i}=2 \mathrm{~km} / 4$ $(k=0,1,2,3))$, the transverse and parallel spatial decay rates can be shown to be the same since the model can be expressed in terms of sums and differences of two identical spin $-\frac{1}{2}$ Ising models. On the other hand, the Goldstone picture suggests that the square root relationship of Theorem 13 should apply to (continuous) rotators for any $D \geqq 2$.

\footnotetext{
3 For some values of the coupling constant.
} 
Acknowledgement. One of the authors (F.D.) is grateful to Prof. Barry Simon and Prof. Henri Epstein for discussions and encouragement; the other author (C.N.) would like to thank Prof. James Glimm for his hospitality at the Courant Institute.

\section{References}

1. Velo, G., Wightman,A.S. (Eds.): Constructive quantum field theory. Berlin-Heidelberg-New York: Springer 1973

2. Guerra,F., Rosen, L., Simon, B.: The $P(\varphi)_{2}$ Euclidean quantum field theory as classical statistical mechanics. Ann. Math. 101, 111-259 (1975)

3. Simon, B., Griffiths, R. B.: The $\left(\varphi^{4}\right)_{2}$ field theory as a classical Ising model. Commun. math. Phys. 33, 145-164 (1973)

4. Richter, V.: Multidimensional local limit theorems for large deviations: Theor. Probability Appl. 3, $100-106$ (1958)

5. Khintchine, A.: Über einen neuen Grenzwertsatz der Wahrscheinlichkeitsrechnung. Math. Ann. 101, 745-752 (1929)

6. Suzuki,M., Fisher, M.: Zeros of the partition function for the Heisenberg, ferroelectric and general Ising models. J. Math. Phys. 12, 235-246 (1971)

7. Lieb,E.H.: The classical limit of quantum spin systems. Commun. math. Phys. 31, 327-340 (1973)

8. Harris, A. B.: Generalizations of the Lee-Yang theorem. Phys. Lett. 33A, 161-162 (1970)

9. Kunz, H.: Location of the zeros of the partition function for some classical lattice systems: Phys. Lett. 32A, 311-312 (1970)

10. Ginibre, J.: General formulation of Griffiths' inequalities. Commun. math. Phys. 16, $310-328$ (1970)

11. Lebowitz, J., Penrose, O.: Private communication

12. Griffiths, R.B.: Rigorous results for Ising ferromagnets of arbitrary spin. J. Math. Phys. 10, $1559-1565$ (1969)

13. Richter, V.: A more precise form of an inequality of S. N. Bernstein for large deviations. In: Selected translations in mathematical statistics and probability, Vol. 4. Providence: Amer. Math. Soc. 1963

14. Lanford, O.E.: Entropy and equilibrium states in classical statistical mechanics. In: Lenard, A. (Ed.): Statistical mechanics and mathematical problems. Berlin-Heidelberg-New York: Springer 1973

15. Glimm, J., Jaffe, A.: The entropy principle for vertex functions in quantum field models. Ann. de l'Inst. H. Poincaré 21, 1-26 (1974)

16. Newman, C. M.: Inequalities for Ising models and field theories which obey the Lee-Yang theorem. Commun. math. Phys. 41, $1-9$ (1975)

17. Hille,E.: Analytic function theory, Vol. II. New York: Ginn 1962

18. Newman, C. M.: Zeros of the partition function for generalized Ising systems. Comm. Pure Appl. Math. 27, 143-159 (1974)

19. Kelly, D. G., Sherman, S.: General Griffiths' inequalities on correlations in Ising ferromagnets. J. Math. Phys. 9, 466-484 (1968)

20. Monroe, J.L.: Correlation inequalities for two-dimensional vector spin systems. Northwestern preprint (1975)

21. Schrader, R.: Private communication

22. Simon, B.: The $P(\varphi)_{2}$ Euclidean (quantum) field theory. Princeton: Princeton University Press 1974

23. Coleman, S.: Private communication via B. Simon

Communicated by A. S. Wightman 
